\title{
ENSEÑANZA DE LA CIENCIA Y EDUCACIÓN EN VALORES
}

\author{
Javier ECHEVERRIA \\ Instituto de Filosofa, CSIC (Madrid) ${ }^{1}$
}

\section{Introducción}

La concepción heredada (received view) restringió excesivamente el ámbito de la filosofía de la ciencia. Primero, porque se centró casi exclusivamente en el conocimiento científico, sin dedicar apenas atención a la práctica científica. Segundo, porque sólo se ocupó del contexto de justificación, es decir del análisis y reconstrucción de los resultados más elaborados de la investigación científica. Ello le llevó a descartar, no sólo el contexto de descubrimiento, sino también lo que atañe a la enseñanza de las ciencias. Tercero, porque separó estrictamente la ciencia y los valores, conforme a la tradición empirista y positivista que escinde los juicios de hecho y los juicios de valor, o si se quiere la razón pura y la razón práctica.

Estas tres restricciones, junto a otras muchas que les son coadyuvantes, han empobrecido el panorama temático de la filosofia de la ciencia en el siglo XX. Durante el último cuarto de siglo han surgido tendencias muy distintas, en el marco de las cuales se sitúa este artículo, que pretende ser una contribución a la filosofia de la práctica científica. Como ya hemos expuesto en una obra anterior $^{2}$, si queremos que la filosofia de la ciencia tenga como referente la ciencia real, y no una idealización del saber científico, hay que criticar esas restricciones

1 «Este artículo ha sido elaborado en el marco del proyecto de Investigación PB-98-0495CO8-01 sobre "Axiología y Dinámica de la Tecnociencia", financiado por el Ministerio de Educación y Cultura.

2 Ver J. ECHEVERrfa, Filosofia de la Ciencia Madrid, Akal, 1995.

ENDOXA: Series Filosificas, n. ${ }^{\circ}$ 14, 2001, pp. 41-59. UNED, Madrid 
y partir de otros supuestos metacientíficos. Por ello proponemos tres tesis contrapuestas a las anteriormente evocadas, a saber:

1. Es precisa una filosoffa de la actividad científica, no sólo del conocimiento científico. La epistemología es tuerta sin una praxiología de la ciencia que, sin desvincularse de la metateoría, sea capaz de analizar y reconstruir la praxis científica, aunque sólo sea parcialmente.

2. La distinción entre los contextos de descubrimiento y justificación es insuficiente, como algunos autores han señalado ${ }^{3}$. La enseñanza de las ciencias plantea problemas filosóficamente relevantes y por ello cabe hablar de un contexto de educación, en el que se desarrollan actividades sin las cuales la ciencia no podría perdurar ni transformar la sociedad.

3. La actividad cientifica en general, y las acciones educativas en particular, están regidas por valores que tienen que ser satisfechos para que dichas acciones puedan ser consideradas como cientificas. Es posible desarrollar una axiología de la ciencia, en cuyo marco hay que analizar los valores vinculados a la enseñanza de las ciencias, es decir al contexto de educación.

El propósito de este artículo consiste en desarrollar esta última tesis y reinterpretar la expresión "educar en valores» desde un punto de vista axiológico, no sólo moral. Incluso ahora, la mayoría de los epistemólogos tienden a pensar que las cuestiones relacionadas con los valores y la ensenanza de las ciencias son menores dentro del ámbito de la filosofía de la ciencia. Entre los pedagogos, en cambio, "educar en valores» es una tarea importante, pero tiene un campo semántico restringido, porque alude ante todo a los valores éticos y morales, o más recientemente a los valores ecológicos. En lo que sigue, trataremos de mostrar la especificidad y la relevancia filosófica de los problemas axiológicos que se plantean en el contexto de educación y propondremos un nuevo marco conceptual para la educación basada en valores.

\section{El contexto de educación y su análisis axiológico}

En La Tension Esencial, Kuhn afirmó que "el contexto de pedagogía difiere del contexto de justificación casi tanto como del contexto de descu-

3 Entre otros, Hanson 1958, TOULmin 1974 y Goldtnan 1983. 
brimiento" 4 . Casi todas las propuestas de Kuhn han sido ampliamente estudiadas por los filósofos de la ciencia, pero esta tesis apenas si ha sido tomada en consideración. A nuestro modo de ver, hay que tomarse en serio la sugerencia kuhniana, distinguiendo un contexto de educación que tiene tanta relevancia filosófica como los otros tres contextos: el de descubrimiento (o innovación, como es preferible llamarlo), el de evaluación (que amplía la propuesta de Reichenbach del contexto de justificación) y el de aplicación, en el que la ciencia se encuentra con problemas específicos, muy distintos a los que surgen en la fase de investigación básica ${ }^{5}$.

Sin enseñanza no hay transmisión del conocimiento y la ciencia no transforma a las sociedades ni a las personas. Para entender un enunciado científico hay que haber aprendido complejos conocimientos, tanto teóricos como prácticos, sin los cuales no hay posibilidad de descubrir, justificar, ni mucho menos de aplicar la ciencia. Asimismo se aprende a llevar a cabo los procedimientos científicos (observación, medida, experimentación, modelización matemática o informática, etc.), por lo que el aprendizaje es una condición sine qua non para la acción científica. Aunque los filósofos de la ciencia han prestado escasa atención a la enseñanza y a la difusión del conocimiento científico, ambas constituyen una componente fundamental de la actividad científica, tomada ésta en toda su extensión. Dicho brevemente: no hay intelección cientifica sin aprendizaje previo. Los procesos de educación científica marcan profundamente nuestra intelección de lo que son las teorías y las prácticas científicas, además de ser esenciales para la constitución de las comunidades científicas, y por ende de los paradigmas científicos. Aunque aquí no vayamos a entrar a fondo en el análisis de esta noción, entendemos la intelección científica como un proceso mental que engloba las capacidades de observar, describir, medir, comprender, operar, experimentar, explicar, interpretar y expresarse adecuadamente.

Desde el punto de vista de la génesis del conocimiento científico en los individuos, la enseñanza de la ciencia es el primer ámbito en donde la actividad científica tiene efectos reales, al transformar las mentes de las personas y

4 T.S. KUHN, La tensión esencial Madrid, F.C.E., 1983, pág. 351. Ver Echeverría 1995, cap. 2, para un estudio más amplio de la distinción y de las defensas y críticas que recibió.

5 Ver Echeverría 1995, cap. 2, para una exposición más amplia de esta distinción entre cuatro contextos. 
estructurarlas conforme a los contenidos, reglas metodológicas y valores que caracterizan a la ciencia. Aquí nos ocuparemos ante todo de los aspectos axiológicos de la enseñanza de la ciencia, en la medida en que aportan una primera vía para el análisis formal de la práctica científica. Pero estudiar el contexto de educación científica requiere otras muchas indagaciones, aparte de esta aproximación axiológica. Expandir el conocimiento científico es el objetivo principal del contexto de educación. De la manera en que ello se haga depende el futuro de la ciencia, así como el modo de inserción del conocimiento en la sociedad. Por ello incluimos también la difusión científica en el contexto de educación.

La educación científica incluye acciones muy diversas, pero cabe distinguir dos acciones básicas, la enseñanza y el aprendizaje, que siempre involucran a seres humanos concretos. Como otras acciones humanas, la docencia y la discencia se desarrollan con ayuda de instrumentos y materiales educativos en los que se representa el conocimiento que ha de ser enseñado y aprendido. En lugar de interesamos por los procesos mentales, cognitivos y comunicativos que se desarrollan al enseñar y aprender las ciencias, aquí nos ocuparemos únicamente de analizar la componente axiológica de las acciones educativas, que es la que resulta relevante para replantear una educación basada en valores. Es preciso analizar los valores subyacentes a la enseñanza de las ciencias si se quiere dar pleno sentido a la expresión «educar en valores». Putnam, Laudan y otros han subrayado la importancia de los valores epistémicos para la ciencia ${ }^{6}$. Si aceptamos que el rigor, la coherencia, la precisión, la generalidad, la verificabilidad, etc., son valores ${ }^{7}$, la expresión "educar en valores científicos" adquiere un sentido muy distinto al que resulta habitual entre los educadores. Por ello la axiología de la ciencia ha de mostrar, en primer lugar, que en las acciones científicas, y en concreto en la enseñanza de las ciencias, intervienen diversos sistemas de valores. A partir de ello la educación basada en valores adquiere un significado mucho más amplio y, a nuestro entender, más pertinente que el habitual. En este segundo apartado desarrollaremos la tesis del pluralismo axiológico en la enseñanza de las ciencias ${ }^{8}$, de modo que, anteriormente, podamos replantear lo que podría ser una enseñanza de las ciencias basada en valores.

6 Ver Putnam 1981 y LaUdan 1984.

7 Así los consideró KUHN en su libro La tensión esencial.

8 Tesis expuesta con mayor amplitud en Echeverría 1995, cap. IV. 
Antes de entrar en ello conviene disponer de unas bases para el análisis axiológico de la enseñanza de las ciencias. Puesto que en este artículo nos ocupamos ante todo de la práctica científica y no de la adquisición de conocimientos, dejaremos de lado los procesos cognitivos que se producen en el curso de la educación y nos centraremos en el análisis de los valores que inciden en las acciones educativas, proponiendo para ello un modelo para analizar dichas acciones en base a nueve componentes. Los procesos de evaluación educativa no sólo se refieren a los resultados, sino también a las restantes componentes de las acciones. El esquema que vamos a usar para el análisis axiológico de dichas acciones desempeña un papel muy importante en todo lo que sigue, porque partiendo de él trataremos de dilucidar la estructura axiológica subyacente a la actividad educativa.

Conforíne a la teoría de la acción científica que hemos propuesto en otro lugar $^{9}$, propondremos el esquema $E$ para analizar axiológicamente las acciones educativas:

E: El agente (docente, discente) A enseria a (aprende de) el agente (discente, docente) B los contenidos $\mathrm{C}$ con los instrumentos I en la situación S, partiendo de las condiciones previas $\mathrm{P}$ y con los objetivos $\mathrm{O}$, dando lugar a los resultados $\mathrm{R}$ y a las consecuencias derivadas $\mathrm{D}$. Todo ello es evaluado conforme al sistema de valores $V$ vigente en el contexto educativo en el que se desarrollan dichas actividades. Educar en valores consiste, en su versión restringida, en incrementar el grado de satisfacción del sistema $V$ por parte de los agentes A y B. En su versión amplia, consiste en incrementar el grado de satisfacción de Vpor parte de cada una de las componentes del esquema $\mathrm{E}$.

Entendemos que este esquema es general, es decir, que tiene validez para analizar axiológicamente cualquier tipo de acción educativa. Por tanto, $E$ nos proporciona un primer análisis de la forma de las acciones educativas y del modo de transformarlas modificando el sistema de valores pertinentes. Antes de explicar con mayor detalle esta manera de concebir la acción de educar en valores, examinemos brevemente las diversas componentes del esquema $E$.

Los agentes A y B son individuos o grupos de individuos. Los verbos «enseñar" y "aprender" pueden ser sustituidos por «educar", «instruir", "formar",

9 J. ECHEVERría "Ciencia, tecnología y valores. Hacia un análisis axiológico de la actividad tecnocientífica", por aparecer en un volumen editado por A, IBARRA y J. A. López CEREzo. 
"difundir", "divulgar" u otros del mismo campo semántico. Las acciones educativas se encadenan unas tras otras, de modo que es preciso haber obtenido unos determinados resultados para poder emprender una nueva acción educativa: por ejemplo para pasar curso, o para entrar en un nivel educativo superior. Los contenidos enseñados o aprendidos son muy diversos: sistemas conceptuales, enunciados, representaciones, imágenes, diagramas, notaciones, técnicas operatorias, resolución de problemas, manejo de instrumentos, acciones a realizar, procedimientos, reglas metodológicas, etc. Los instrumentos docentes también son variados, y van desde el habla a la escritura, pasando por la lectura, los libros, las ilustraciones, los mapas, los diagramas, las fórmulas, las tablas, los instrumentos de medida y de cómputo y, más recientemente, los ordenadores. Por situación docente aludimos al contexto social y a los escenarios concretos en que se desarrollan los procesos educativos: épocas, culturas, instituciones, constricciones sociales, políticas, económicas, idiomáticas o religiosas, relaciones entre los agentes, aulas, laboratorios docentes, bibliotecas, museos, etc. De dichas actividades educativas surgen resultados, por ejemplo la adquisición de nuevos conocimientos y experiencias, que pueden ser positivos o negativos (éxito y fracaso escolar o universitario). Cada agente $A$ y $B$ tiene que mostrar que ha interiorizado los contenidos que son enseñados, demostrando que sabe la teoría y también que sabe hacer, es decir, que tiene una competencia teórica y práctica en el uso de los instrumentos docentes y en la comprensión de los contenidos. A partir de ello podrá ser reconocido o rechazado como posible miembro de una comunidad científica. Además, las acciones educativas son intencionales, están regladas y se orientan al logro de determinados objetivos. Por otra parte, siempre tienen en cuenta el punto de partida, es decir, las condiciones iniciales en las que los agentes docentes, y sobre todo los discentes, participan en dichos procesos. Una de las características más peculiares de las acciones educativas consiste en su adecuación a la edad y al nivel cognitivo de alumnos y estudiantes. Por último, no hay que olvidar que a lo largo de esos procesos educativos se producen una serie de consecuencias adicionales, que van más allá de los objetivos inicialmente previstos: por ejemplo, se desarrollan procesos de socialización, se aprende (o no) a trabajar en equipo, surgen vocaciones ( $\mathrm{y}$ rechazos) intelectuales, se producen identificaciones, etc. En la enseñanza de las ciencias no sólo hay que evaluar el logro de los objetivos propuestos, sino también los beneficios y daños adicionales. Para los propósitos de este artículo, lo importante es tener en cuenta que todas y cada una de las diez componentes son evaluadas conforme a sistemas de valores 
previamente existentes, sin perjuicio de que dichos sistemas de valores no sean estáticos, sino dinámicos, pudiendo modificarse a lo largo de los procesos educativos. La axiología de la educación tiene por objeto analizar los procesos de evaluación de esas diez componentes y, en su caso, proponer mejoras, es decir, incorporar nuevos valores, así como incrementar o menguar el grado de satisfacción de valores específicos.

Este esquema inicial, con las implementaciones y precisiones correspondientes, vale también para los procesos de difusión y divulgación científica. Por ello lo adoptaremos como un primer modelo para analizar las actividades que tienen lugar en el contexto de educación. Desde la Revolución francesa, la enseñanza de las ciencias está socialmente regulada y posee sus propias técnicas de presentación, justificación, valoración y aplicación, que no tienen por qué coincidir con las usadas en los restantes contextos de la actividad científica. No es lo mismo evaluar un proceso de aprendizaje que un proyecto de investigación o una aplicación concreta de una teoría o innovación científica. En el contexto de educación siempre hay una mediación social que delimita los conocimientos y habilidades a adquirir, las técnicas pedagógicas y los sistemas de evaluación. Ello es particularmente claro a partir del establecimiento de la enseñanza obligatoria, que exige la adquisición de unas nociones científicas elementales por parte de todos los ciudadanos de un país. El contenido de lo que se ha de enseñar ha sido fijado previamente, en forma de planes de estudio, al igual que los escenarios, los agentes y los instrumentos docentes.

Uno de los objetivos básicos de la enseñanza de la ciencia es la adquisición por parte de los estudiantes de representaciones mentales adecuadas de conocimientos científicos previos. Dichas representaciones no sólo son lingüísticas: no basta con poseer los conceptos o saberse de memoria las leyes básicas de una determinada teoría, sino que hay que haber interiorizado el por qué de dichas teorías, así como las técnicas de escritura, observación, medición, cálculo y experimentación que van ligadas a la misma. En esta época de formación se prefigura la adscripción del futuro científico (o profesional) a uno u otro paradigma y comunidad científica. Es importante subrayar que la construcción de representaciones mentales nunca es una actividad exclusivamente individual, sino que está profundamente mediatizada y controlada por la sociedad. Esta determina, al menos en la enseñanza reglada, los contenidos a enseñar, el orden de su presentación y las actividades prácticas a realizar. A continuación evalúa el nivel del individuo a través de sus agentes docentes, dilucidando su mayor o menor competencia y aptitud, al par que corrigiendo, motivando y, en general, 
normalizando las representaciones mentales que el sujeto individual se haya hecho de las teorías. El ámbito por excelencia para la ciencia normal kubiniana es el contexto de educación. Las diversas comunidades científicas siempre pugnan por tener agentes que difundan activamente sus paradigmas en el ámbito docente. Los procesos de cambio en el contexto educativo suelen resultar mucho más lentos y difíciles de llevar a cabo que los cambios científicos en el contexto de innovación. Estos últimos son los únicos que suelen ser estudiados por los filósofos de la ciencia, lo cual constituye una clara insuficiencia: los procesos de cambio en el contexto de educación siempre son ilustrativos de los grandes cambios científicos, pero entendidos éstos como cambios sociales, no solamente como transformaciones en el seno de la propia comunidad científica.

Conviene tener presente, además, que la difusión y la divulgación científica (a través de revistas, videos, programas de radio y televisiones, colecciones de libros de bolsillo, imágenes tecnocientíficas, etc.) ha de ser incluida en este primer ámbito de la actividad científica. Nuevamente se repite el proceso, pero esta vez para un número mucho mayor de destinatarios: las teorías y los descubrimientos se presentan en forma simplificada y accesible, recurriéndose a representaciones ad hoc. Incluso los medios de comunicación dedican suplementos y programas especiales para esta labor de divulgación científica, que es la que genera una imagen social de la investigación, de las teorías y del progreso científico. La divulgación científica ha solido ser desdeñada por los filósofos de la ciencia como ámbito de estudio. Sin embargo, es una componente importante de la actividad científica en general, y por ello la incluimos en el contexto de educación.

\section{Análisis formal de las acciones educativas}

En el apartado anterior hemos propuesto un modelo para analizar las acciones educativas, basado en diez componentes, Veamos brevemente lo que aporta el modelo $\mathrm{E}$ a la hora de analizar acciones educativas concretas. Estas son muy diversas, por lo que elegiremos unos cuantos ejemplos canónicos, con el fin de mostrar cómo se aplica el modelo anterior al análisis de dichas acciones. Sean pues las acciones educativas:

1) Un(a) maestro(a) enseña matemáticas (física, química, etc.) a estudiantes en un aula. 
2) Un niño/a lee un libro de texto o consulta una enciclopedia en un CD-ROM educativo.

3) Un profesor/a lleva a sus alumnos a visitar un museo de la ciencia.

4) Un profesor/a examina a sus alumnos/as a fin de curso.

5) Un colegio hace públicas las calificaciones de sus estudiantes a fin de curso.

6) Una revista de divulgación difunde algún descubrimiento científico.

7) Un profesor/a es evaluado/a por su actividad docente.

8) Hay un cambio de plan de estudios.

9) Un libro es homologado como obra de texto.

10) Se crea una nueva institución educativa.

Podrían proponerse otros muchos ejemplos, pero los diez anteriores pueden bastar para aplicar el esquema $E$ al análisis axiológico de las acciones educativas. A la hora de valorar esas diez acciones no basta con esos enunciados breves, sino que hay que explicitar las diez componentes del esquema $E$, o al menos la mayor parte de ellas. De lo contrario no se traslucen los diversos sistemas de valores involucrados en las acciones educativas.

Consideremos la acción primera. El agente $A$ (maestrola) ha sido habilitado socialmente para ejercer su función y para ello ha tenido que superar satisfactoriamente una serie de pruebas previas. Es decir, todo agente educativo ha sido evaluado en función de un subsistema de valores $V_{a}$ para poder llevar a cabo la acción de enseñar. Otro tanto cabe decir del alumno $B$, quien ha tenido que superar una serie de pruebas y requisitos para acceder al aula en la que aprende. Ello garantiza una parte de las condiciones iniciales (cognitivas) por parte de alumnos y profesores, siendo un requisito axiológico previo para que la escena del aula se produzca. Los contenidos que se enseñan suelen estar prefijados por los planes de estudio en función del nivel educativo del que se trate. Otro tanto cabe decir del aula, del colegio, de los instrumentos docentes que se utilizan y de los objetivos del curso y de la clase, habitualmente prefijados y homologados. Todo ello remite a la situación, es decir al sistema institucional en el que la acción docente se enmarca, el cual refiere a su vez a la cultura, a la época, a los medios económicos y humanos con que se cuenta, al estado de la ciencia en ese momento, etc. Aceptando que todas esas componentes han sido 
previamente valoradas en función de diversos subsistemas $V_{p}$ la acción como tal produce unos resultados y unas consecuencias adicionales, que a su vez son evaluadas. En resumen, para cada componente $e_{i}$ del esquema $E$ hay un subsistema $V_{i}$ de valores pertinentes $v_{i j}$, algunos de los cuales se solapan entre un subsistema y otro, mientras que otros son específicos para cada componente. Este primer análisis axiológico de la acción 1 muestra la existencia de un complejo sistema de valores $V$, estructurado en subsistemas $V_{p}$ cada uno de los cuales se aplica a la evaluación de cada una de las diez componentes $e_{i}$ En conjunto, el proceso de evaluación puede ser representado como $V_{i}\left(e_{i}\right)$, o, teniendo en cuenta que $V_{i}=\left\{v_{i j}\right\}$ la evaluación completa de una acción educativa ofrece como resultado la matriz $v_{i j}\left(e_{i}\right)$. Conviene advertir que esa matriz de evaluación no es estática y que, por lo general, los procesos de valoración no funcionan en base a criterios de maximización de la función de utilidad subyacente a dicha matriz, sino que son procesos de criba, basados en la superación de determinados umbrales de satisfacción de los diversos valores vij. El análisis axiológico de los procesos educativos no tiende al objetivo de que el mejor profesor enseñe al mejor alumno los mejores contenidos en el colegio mejor y con los mejores instrumentos docentes. Muy al contrario. Dado un cierto nivel de satisfacción mínima de ciertos valores nucleares (requisitos axiológicos), se trata de incrementar el grado de satisfacción de esos valores, más que de maximizarlos. Las diez componentes del sistema $\mathrm{E}$ no están aisladas, sino que constituyen un sistema, en cuyo marco es posible llevar a cabo la acción docente, así como evaluarla y, en su caso, mejorarla.

Esta propuesta de análisis de la acción 1 puede ser repetida en el caso de las otras diez acciones mencionadas y, en general, en las diversas acciones educativas que podamos considerar. Lo importante es que el incremento, directo o indirecto, del conocimiento en las mentes de los estudiantes siempre es resultado de una acción con diversas componentes, cada una de las cuales puede ser evaluada ( $\mathrm{y}$ mejorada). El fracaso escolar, por ejemplo, puede ser atribuido al estudiante, pero también puede provenir del profesor, de los contenidos elegidos, de los instrumentos usados o de la inadecuación de los objetivos. La axiología que proponemos es sistémica y por ello también la noción de "educar en valores" ha de ser analizada desde este punto de vista sistémico. Para aumentar el grado de satisfacción de un valor determinado en una acción educativa no hay que actuar sólo sobre los agentes: también hay que intervenir sobre los contenidos, los escenarios, los instrumentos y las condiciones iniciales. 


\section{Los valores en el contexto de educación}

Partiendo del modelo propuesto para el análisis axiológico de las acciones educativas, se trata ahora de dilucidar cuáles son los valores relevantes en el caso de la enseñanza de las ciencias. Muchos de ellos son comunes a los del resto del sistema educativo, pero también hay algunos especificos en el caso de la enseñanza de las ciencias, como veremos a continuación. Puesto que del primer tipo de valores ya nos hemos ocupado anteriormente ${ }^{10}$, nos centraremos ahora en los valores específicos de la enseñanza de las ciencias.

Empecemos por un argumento que puede parecer obvio. Para enseñar una ciencia es preciso que el agente docente $A$ tenga la formación científica y pedagógica pertinente. ¿Cómo se acredita esto? Superando una serie de pruebas que constituyen otros tantos requisitos axiológicos para los agentes docentes posibles. Se exigirá, por ejemplo, ser maestro, licenciado o doctor, según los diversos niveles educativos. Pero este requisito puede ser suavizado en función de la situación, de los medíos, del contexto social e institucional, etc. Lo importante es que el nivel de competencia científica y pedagógica de los agentes docentes vaya incrementándose progresivamente, sea mediante la exigencia de titulaciones, mediante cursos de formación o reciclaje o a través de los correspondientes procesos de control y evaluación. En cualquier caso, cada agente educativo ha de haber pasado pruebas de evaluación de muy diversa índole, tanto antes como después de haber iniciado su labor docente. Educar en valores implica incrementar el nivel profesional del profesorado, su dedicación, su motivación, su capacidad de adaptación a los cambios metodológicos, epistemológicos y pedagógicos, etc. $Y$ para ello es preciso tener en cuenta que enseñar no es lo mismo que investigar o que aplicar los conocimientos científicos. Los criterios para evaluar al profesorado no coinciden con los que se aplican en el contexto de investigación o en el de aplicación.

Otro tanto cabe decir de los instrumentos docentes, por ejemplo de los libros de texto, de los laboratorios, de las bibliotecas, de las salas informáticas o de las técnicas para el trabajo de campo. La evaluación de la componente I difiere grandemente de la componente $A$, pero tampoco puede ser desligada de ella. La claridad, la sencillez, el rigor, la sistematicidad, la accesibilidad, la facilidad de uso, la modernidad, etc., son algunos de los valores relevantes en el caso de

10 Ver Echeverría 1995, apartado IV.3. 
la componente $I$, pero en muchos casos han de ser analizados en conexión a los agentes $A$ que utilizan esos instrumentos y a su aptitud y preparación para usarlos. El ejemplo de los ordenadores en la enseñanza resulta suficientemente ilustrativo. De poco sirve mejorar los instrumentos docentes en las escuelas sin una acción paralela sobre el profesorado que los ha de usar. Y no hay que olvidar que tanto en un caso como en otro hay valores económicos que también intervienen: desde el coste de los salarios y retribuciones hasta la rentabilidad de las inversiones que se hagan en nuevos medios docentes, pasando por el principio de igualdad de oportunidades en el uso de dichos instrumentos.

Por supuesto, es fundamental evaluar los contenidos que se van a enseñar, sea por su grado de cientificidad, de actualidad, de utilidad social o de adaptación a la cultura en la que se imparten. Si aceptamos, por ejemplo, que la multiculturalidad de los contenidos es un valor en el contexto de educación, aquellas acciones que tiendan a adecuar los contenidos epistémicos y las reglas metodológicas de la ciencia a la cultura en la que se enseñan serán una forma más de educar en valores. No es lo mismo educar que formar para una profesión o para encontrar un puesto en el mercado de trabajo. Los sistemas de valores relevantes para una u otra acción son distintos, aunque puedan tener un núcleo epistémico común.

Este último ejemplo nos conduce a un debate de gran importancia, relativo a los objetivos de las acciones educativas. Las teorfas dominantes sobre la racionalidad cientifica han tendido a identificar esa forma de racionalidad con algunos objetivos generales de la ciencia, como la búsqueda de la verdad (o de la verosimilitud), el incremento de la contrastación empírica, de la preductividad, del carácter explicativo de las teorías científicas, etc. En el contexto de educación, la racionalidad de las acciones no es explicable en base a estos criterios, precisamente porque intervienen otros sistemas de valores que modulan y atemperan los pretendidos objetivos generales de la ciencia en otros contextos. Por ejemplo, nadie en su sano juicio pretende enseñar a niños y niñas las teorías científicas tal y como éstas son, con todo su nivel de complejidad y exactitud. La accesibilidad del conocimiento científico y la posibilidad de que éste sea entendido al nivel cognitivo en el que estén los alumnos prima netamente sobre el rigor y la exactitud a la hora de exponer dichos contenidos. Ello no equivale a decir que los criterios y objetivos recién mencionados sean irrelevantes en el contexto de educación. Lo que ocurre es que se ven contrapesados por otros objetivos, de modo que funcionan como valores a tener en cuenta, no como metas definitorias de la racionalidad de los procesos educativos. 
Tenemos con ello otro argumento a favor de la gradualidad en la satisfacción de los valores y objetivos epistémicos y en contra de las teorías maximizadoras, las cuales, caso de que fueran aplicadas en el contexto de educación, conducirían a auténticos absurdos pedagógicos.

Pasemos a otra componente de las acciones educativas, a saber, las consecuencias derivadas de las mismas. Una de las más típicas es el rechazo a determinadas materias, contenidos o instrumentos educativos, derivado de una introducción inadecuada de los mismos. Este riesgo ha de ser especialmente evaluado, y por ello la última componente del esquema $E$ ha de ser tenida muy en cuenta, en lugar de evaluar los resultados únicamente en función de los objetivos prefijados. Una teoría de la acción educativa racional ha de estar basada en un análisis detallado de las acciones educativas, en el que se incluyan todos sus componentes relevantes, así como los sistemas de evaluación específicos para cada uno de ellos. De nuevo encontramos un argumento a favor de distinguir diversos subsistemas de valores $V_{p}$ en lugar de priorizar únicamente el análisis de los resultados, por ejemplo en términos de eficacia educativa, midiendo el porcentaje de éxitos finales sin tener en cuenta los fracasos intermedios y los costes que de ellos se derivan.

Podríamos proseguir nuestra argumentación con las diez componentes del esquema $E$, mas lo dicho hasta ahora puede bastar para ilustrar el sentido de nuestra tesis. En lugar de pensar que la ciencia se rige por valores internos, epistémicos o cognitivos, como han afirmado la mayorla de los epistemólogos que se han ocupado de estas cuestiones, desde el momento en que nos interesemos en el contexto de educación como objeto de la filosofia de la ciencia, y más si lo hacemos desde una perspectiva axiológica, es preciso tener en cuenta que en las acciones educativas se ven involucrados sistemas de valores muy diversos, que deben ser analizados adecuadamente. Hasta el momento hemos propuesto un análisis basado en las diez componentes del esquema $E$. Pero ya advertimos que los subsistemas $V_{i}$ que resultan pertinentes para evaluar cada componente $e_{i}$ no son estancos, sino que tienen puntos comunes entre sí, y también diferencias. Por poner un ejemplo sencillo: la claridad es un criterio de evaluación que resulta pertinente para los agentes, los instrumentos docentes, los contenidos y los resultados, como mínimo. Ello no equivale a decir que signifique lo mismo en uno y otro caso. Y otro tanto, cabría decir de la fiabilidad, de la coherencia o de la precisión. En lugar de basar la axiología de la ciencia en una teoría del significado, interpretando los valores como ideas, o incluso como conceptos 
cuyo contenido es o debería ser unívoco, es preciso un análisis más fino de los valores vinculados a la actividad científica, y en concreto a las acciones educativas. La concepción semántica en filosofía de la ciencia ofrece al respecto una alternativa analítica. Si considerarnos que un valor viene definido por la clase de modelos empíricos que lo satisfacen en cierto grado, podemos analizar los diversos subsistemas de valores en términos conjuntistas, como clases de clases de modelos, siendo cada valor una clase modelos. La existencia de protocolos de evaluación para las actividades docentes (baremos, pruebas objetivas) garantiza un sistema de medida del grado de satisfacción de cada valor por parte de un sistema empírico concreto, es decir, por cada acción educativa. Puesto que, como hemos mostrado en el ejemplo de la acción 1, casi siempre hay protocolos de evaluación ponderada para cada una de las componentes de una acción educativa, dichos protocolos de evaluación permiten establecer los valores concretos de la matriz $v_{i j}\left(e_{i}\right)$ en cada acción concreta.

Frente a una axiología atomista y estática, pensamos que la axiología de la ciencia ha de ser sistémica y dinámica, siempre atenta a los cambios que se suscitan dentro de cada subsistema de valores, y en conjunto en el sistema V. Ello implica una axiología empírica, basada en el estudio de casos concretos, pero disponiendo de un, modelo formal común, como el propuesto en el esquema $E$, o en algún otro esquema más perfeccionado. La axiología que proponemos, por tanto, no es normativa, pero tampoco se limita a ser descriptiva, o puramente analítica. Como dijimos al principio, el análisis empírico y descriptivo es la primera fase de la misma, pero no la única.

Veamos por último en qué sentido la axiología que proponemos, sin ser normativa, puede llegar a ser transformadora de la actividad científica, y en concreto de las acciones educativas.

\section{Educar en valores}

Conforme hemos ido anticipando a lo largo de este texto, educar en valores no supone sólo proporcionar una buena formación ética o moral, sino que afecta a los diversos subsistemas de valores que están implicados en las acciones educativas. No hay duda de la importancia de valores como la honestidad y la veracidad en el contexto de educación, pero las cuestiones axiológicas no sólo son morales, sino que también pueden ser epistémicas, tecnológicas, económicas, 
ecológicas, políticas, jurídicas, culturales, estéticas e incluso militares ${ }^{11}$. En ello radica la peculiaridad mayor de la enseñanza de las ciencias, debido a que la actividad cientifica en general está vinculada a todos esos subsistemas de valores.

Veámoslo con mayor detalle. En el contexto de justificación suele exigirse que las teorías científicas sean verosímiles, precisas, rigurosas, coherentes, predictivas, explicativas, contrastables empiricamente, etc. Este conjunto de valores forman parte del núcleo axiológico de la investigación científica. Una ensefianza de las ciencias basada en valores no debe preocuparse únicamente por transmitir conocimientos, sino que además ha de ser capaz de que los estudiantes interioricen esos valores epistémicos, y ello poco a poco, por fases. Lo que se le pide a un investigador o a un profesional no se le puede exigir a un estudiante, pero en la medida en que los procesos educativos traten de formar profesionales para la ciencia, es preciso que las acciones educativas tengan como uno de sus objetivos la transmisión de esos valores epistémicos, no sólo de los contenidos teóricos o de los procedimientos prácticos para actuar científicamente. Cabe decir incluso que la interiorización de los valores específicos de la ciencia es una parte esencial en la formación de cientificos, debido a que esos valores son constitutivos de lo que Snow llamó la segunda cultura. En la medida en que los estudiantes sean más precisos y rigurosos a la hora de expresarse o de actuar (observando, midiendo, experimentando...), o en que sus ideas e hipótesis sean más verosímiles, coherentes, contrastables, explicativas, etc., los procesos de formación científica tendrán mayor éxito, independientemente de los contenidos que se estén enseñando. La formación en valores epistémicos es una de las maneras de educar en valores.

Mas no la única. Tanto a la hora de investigar como de aplicar sus conocimientos en el futuro, los científicos se van a encontrar con problemas técnicos, económicos, ecológicos y jurídicos, e incluso con problemas políticos, culturales, estéticos y militares. Suscitar la preocupación por este tipo de cuestiones axiológicas y darles una adecuada formación al respecto es tan importante como proporcionarles una buena formación moral y epistémica. Por ello afirmamos que la educación en valores axiología de la educación científica ha de habérsela con conflictos y contradicciones, Cabe incluso afirmar que los conflictos de

11 Para un estudio más amplio de los diversos tipos de valores vigentes en la actividad científica, ver J. ECHEVERRIA: "Tecnociencia y sistemas de valores", por aparecer en un volumen editado por J. M. SÁNCHez Ron y L A. López Cerezo. 
valores son uno de los objetos de estudio más importantes para la axiología de la ciencia, y ello no sólo en el contexto de educación, sino también en los de innovación, evaluación y aplicación. Los propios procesos de evaluación suelen ser conflictivos, y este hecho, mil veces comprobado en la enseñanza, es una consecuencia de la estructura del sistema $V$, con sus diversos subsistemas, y de la propia especificidad de los valores como objeto de estudio, al no haber un valor sin su contrario, y al ser $V$ un sistema dinámico, no estático.

Pongamos un ejemplo. Casi todos los autores que se han ocupado de la praxis de los científicos señalan que ésta es cooperativa, por una parte, pero también competitiva. Bruno Latour es uno de los que más ha insistido en este punto, al afirmar que los científicos pugnan ante todo por el reconocimiento ${ }^{12}$. Para lograrlo, es preciso trabajar en equipo, sobre todo en el caso de la ciencia contemporánea. Pero la capacidad de trabajar en equipo no excluye la de competir dentro del mismo equipo, sino al revés: es la otra cara de la misma moneda. En la medida en que la enseñanza de las ciencias no sólo sea teórica, sino también práctica, es preciso educar a los estudiantes tanto para cooperar como para competir. Y aparte de la oposición cooperación/competición podríamos mostrar otros ejemplos de valores opuestos entre sí, y que sin embargo deben ser satisfechos en un cierto grado. Los científicos han de saber ser autónomos e independientes, pero también han de aprender formas de heteronomía y de dependencia.

De las consideraciones anteriores se deriva una consecuencia interesante para el esquema $E$ que hemos propuesto en este artículo. Las acciones educativas no sólo implican diversos subsistemas de valores, sino también valores contrapuestos. Ello equivale a decir que en las evaluaciones $v_{i j}\left(e_{i}\right)$ no sólo tendremos resultados positivos, sino también negativos. Es otro de los motivos por los que contraponemos la noción de criba axiológica a la de maximización de una función de utilidad. En lugar de pensar en que hay que maximizar los valores y minimizar los disvalores, una axiología racional de la ciencia (y de la educación científica) ha de basarse en las nociones de umbrales máximos (para los disvalores) y mínimos (para los valores). La axiología puede intervenir en los procesos de evaluación, sin por ello ser normativa, precisando esos umbrales máximos y mínimos para los diversos valores $v_{i j}$, pertenecientes a subsistemas diferentes, así como proponiendo fijar, a continuación, nuevos umbrales máximos y mínimos.

12 Ver, por ejemplo, su obra Ciencia en acción (1987). 
La idea subyacente a esta propuesta es muy sencilla, y se entenderá bien retornando el ejemplo de la acción 1: Un(a) maestro(a) enseña matemáticas (física, química, etc.) a estudiantes en un aula. Si ese maestro tiene una formación mejor (tanto desde el punto de vista científico y pedagógico como por su formación en valores), si el aula satisface en mayor grado diversos requisitos (por ejemplo ambientales), sí el número de estudiantes presentes en el aula se reduce, pero sin caer por debajo de un umbral mínimo, si los medios económicos de la institución educativa aumentan, si la formación previa con la que llegan los estudiantes es mejor, si los instrumentos docentes se actualizan en la medida de lo posible, etc., estamos ante un ejemplo de mejora de una acción educativa por incremento de los umbrales mínimos para los valores y decremento de los umbrales máximos para los disvalores.

Algunos de quienes lean este artículo pensarán: ¡pero sí es lo que trata de hacer cualquier educador! Pues si es así, el esquema E que hemos propuesto vale para el versa sobre diversos subsistemas de valores, y no sólo sobre valores morales. Incluso los valores estéticos tienen importancia para la educación de los científicos: no es lo mismo una demostración matemática o un programa informático que sean claros y elegantes (además de ser precisos, rigurosos, coherentes, eficaces, etc.) a que los pasos deductivos o iterativos sean oscuros, incomprensibles, ilegibles, etc. Y lo dicho para las demostraciones vale también para las teorías, las hipótesis, los experimentos y las bases de datos. En la medida en que las acciones de los estudiantes satisfagan progresivamente más valores, y en mayor grado, se producirá un avance educativo y formativo.

Dichas las cosas así, pueden parecer obvias, pero lo cierto es que no suele razonarse en estos términos a la hora de plantear la educación, la formación o incluso la difusión científica basada en valores. Detengámonos en el ejemplo de la divulgación científica, porque es suficientemente ilustrativo. En muchas ocasiones, lo que prima a la hora de difundir los descubrimientos científicos, por ejemplo a través de los medios de comunicación, es el sensacionalismo o el pintoresquismo. Si los periodistas científicas hubieran recibido una formación en valores, incluyendo los valores epistémicos, en sus publicaciones se valoraría el rigor, la precisión, la contrastación de las noticias, el interés social de las mismas, etc. Podrá decirse que esto forma parte de la deontología periodística en general, y que no afecta sólo a la divulgación científica. Así es. Lo importante es tener en cuenta que la formación en valores, en el sentido amplio en que aquí utilizamos esta expresión, es precisa también para quienes enseñan, forman o divulgan la ciencia. 
En segundo lugar, las obviedades anteriores tienen no pocas consecuencias filosóficas. Mencionaremos tres. Primero, los agentes educativos y sus acciones han de satisfacer esos mismos valores, porque de otro modo dificilmente se interiorizarán los valores relevantes para la actividad científica, a no ser por la vía de la crítica y el rechazo a los educadores, formadores y divulgadores. Por cierto que esto último ocurre con frecuencia. Segundo, y más importante, la noción filosófica adecuada es la satisfacción gradual de los diversos valores, noción que se opone a la de maximización de un valor único. El monismo axiológico no es un marco adecuado para educar en valores a los científicos, contrariamente a quienes piensan que la racionalidad cientifica consiste ante todo en maximizar un valor, sea éste la verdad, la verosimilitud, el rigor, la predictibilidad o la contrastación empírica. Todos estos valores son relevantes para la enserianza de las ciencias, pero ninguno de ellos tiene la primacía sobre los demás o, si en algún momento la tiene, en otro puede dejar de tenerla. Por ello la axiología que propugnamos es gradualista y dinámica. Tercero, en los procesos educativos no sólo hay que atender a los valores objetivos, sino también a los subjetivos. En lugar de perderse en el laberinto de la controversia sobre la objetividad o subjetividad de los valores, en el contexto de educación hay que tener claro que ambos tipos de valores son significativos.

En tercer lugar, en el contexto de educación hay conflictos de valores. Sería vano imaginar un sistema $\mathrm{V}$ ideal de valores para el contexto de educación, porque no hay valores sin contravalores, y son muchas las ocasiones en que al exigir la maximización de un valor se generan consecuencias indeseables en otros ámbitos axiológicos. Dichos conflictos de valores pueden manifestarse en las personas, pero también entre las instituciones y las culturas. Los valores epistémicos que caracterizan al conocimiento científico han de acoplarse y armonizarse, en la medida de lo posible, con otros sistemas de valores, precisamente porque los procesos educativos están mediatizados socialmente. A diferencia de otras ramas de la filosofia de la ciencia, la análisis axiológico de las acciones educativas, sin perjuicio de que pueda encontrarse uno mejor. Introducir dicho esquema en el contexto de educación, que está estrechamente vinculado al contexto de evaluación, sena una de las acciones que la axiología de la ciencia que propugnamos podría hacer en los ámbitos educativos, o cuando menos en algunos de sus escenarios.

Precisamente por ello la axiología de la ciencia, tendiendo a ser analítica, empírica e incluso formal, puede intervenir en la actividad científica, sin llegar por ello a ser normativa. 


\section{Bibliografía}

AGAZZI E. (1996): El bien, el mal y la ciencia. Las dimensiones éticas en la empres a cientifico-tecnológica Madrid, Tecnos.

Bunge M. (1988): Ética y ciencia, Buenos Aires, Siglo XX.

ECHeverrfa J. (1995): Filosofia de la Ciencia, Madrid, Akal.

ECHEVERRfA J. (1998): "Ciencia y valores: propuestas para una axionomía de la ciencia»en Pascual F. Martínez Freire (ed.), Filosofia actual de la ciencia, Contrastes, Málaga, Universidad de Málaga, 175-194.

HACKING I. (1982): Representing and Intervenin Cambridge, Cambridge Univ. Press.

Hanson N. (1958): Pattems of Discovery, Cambridge, Cambridge Univ. Press.

Kunn T. S. (1977): The Essential Tension, Chicago, Univ. of Chicago Press.

LaTour B. (1992): Ciencia en Acción, Barcelona, Labor, traducción de Latour 1987.

LAUdAN L. (1984): Science and Values Cambridge, Harvard Univ. Press.

Putrfain H. (1981): Reason, Truth and Histo, Cambridge, Cambridge Univ. Press.

RESCHER N. (1999): Razones y Valores en la Era cientifico-tecnológic, a Barcelona, Paidós.

Toulmin S. (1974): Scientific Strategies and Historical Change, Boston, Boston Studies in the Philosophy of Science, vol. 11. 